\title{
SUPPORTING INFORMATION \\ Reprogrammable 3D Shaping from Phase Change Microstructures in Elastic Composites
}

Heng Deng ${ }^{\#}$, Xianchen Xü ${ }^{\#}$, Cheng Zhang, Jheng-Wun Su, Guoliang Huang*, Jian Lin ${ }^{1,2,3 *}$

${ }^{1}$ Department of Mechanical and Aerospace Engineering

${ }^{2}$ Department of Electrical Engineering and Computer Science

${ }^{3}$ Department of Physics and Astronomy

University of Missouri, Columbia, Missouri 65211, USA.

*Emails: linjian@missouri.edu or huangg@missouri.edu

\# These authors contributed equally to this work 


\section{Supplementary Figures \& Caption}

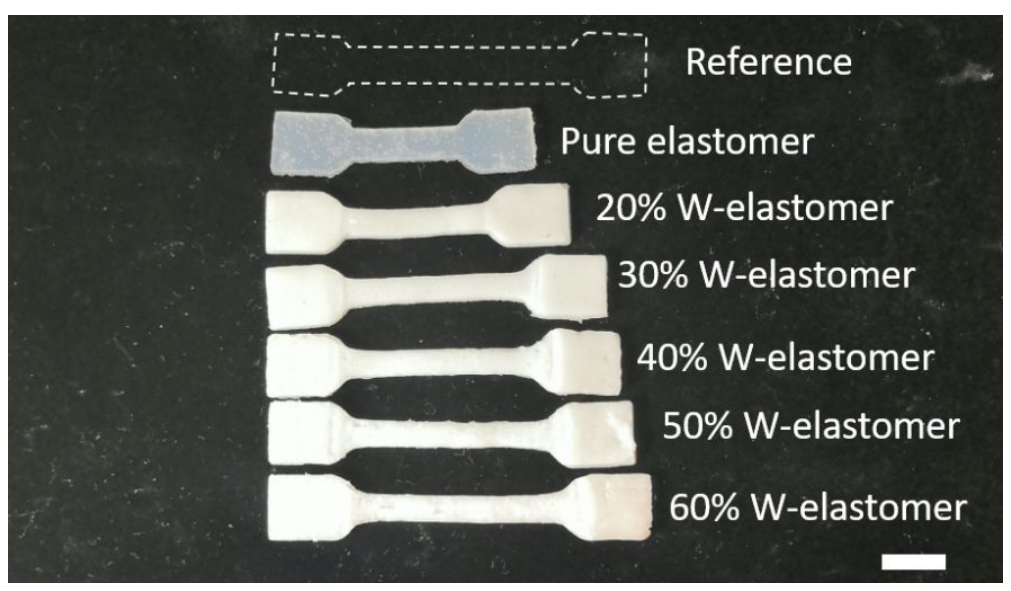

Figure S1. Photograph showing different residual strains remained in the W-silicone composite sheets with wax of different mass ratios. These $\mathrm{W}$-silicone samples were pre-stretched by a strain of $100 \%$. Then these stretched W-silicone were heated at $100{ }^{\circ} \mathrm{C}$. After W-silicone composites were cooled down, the applied external stress was removed. Scale bar: $1 \mathrm{~cm}$.
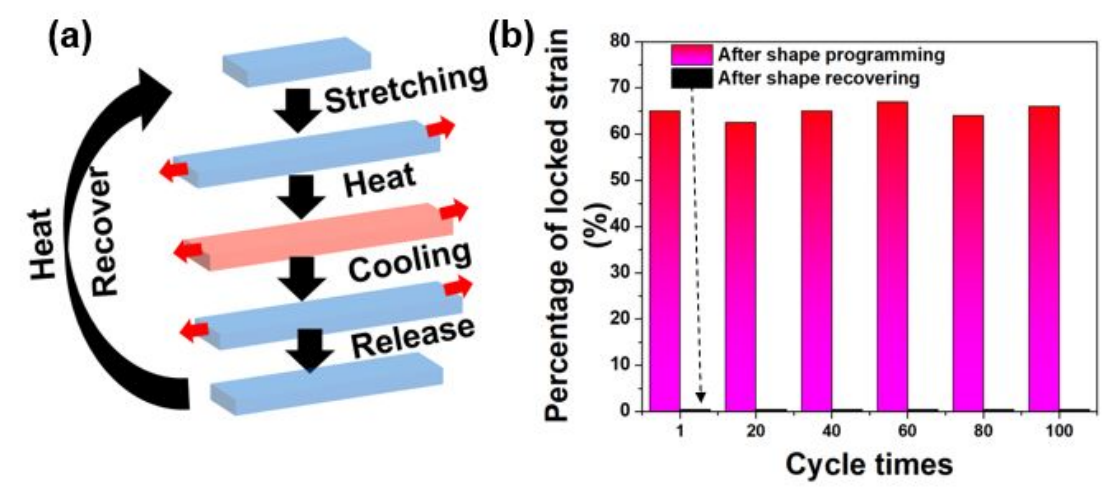

Figure S2. (a) Schematic of a programming process for cycling test of residual strain in a W-silicone composite. (b) Evolving of residual strain as a function of cycling times. The residual strain in the Wsilicone composite is consistent after 100 cycles of the programming process. And the residual strain can be erased by heat, making the $\mathrm{W}$-silicone sheet recover to the original state. 
(a)

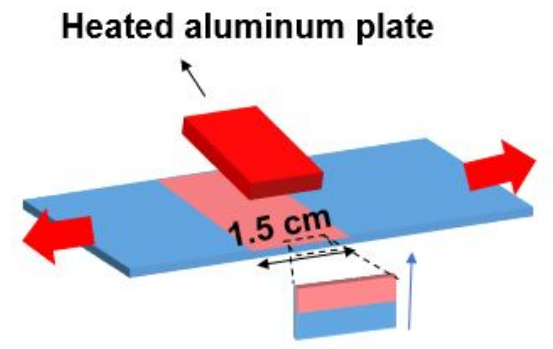

(b)

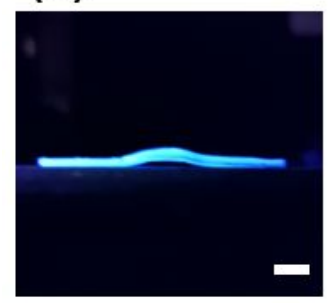

$1 \mathrm{~s}$
Short time

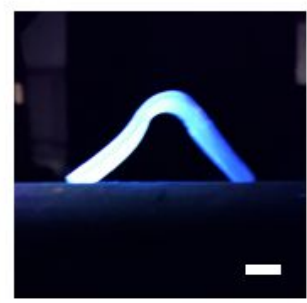

$2 \mathrm{~S}$

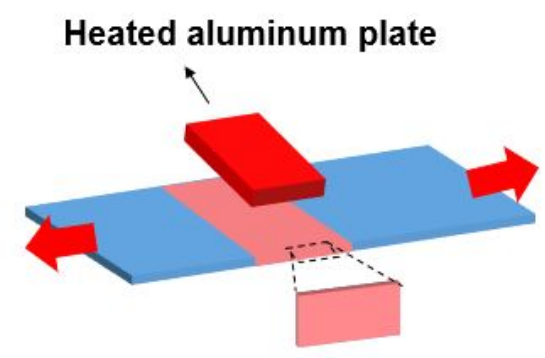

Long time

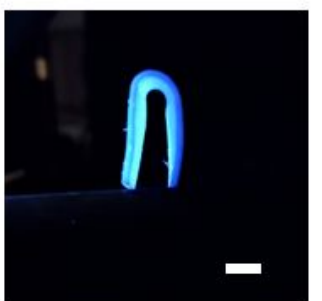

$3 \mathrm{~s}$

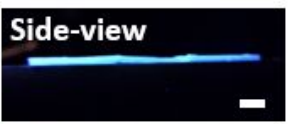

Top-view

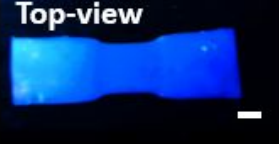

$4 \mathrm{~S}$

\section{Heating time}

Figure S3. (a) Schematic showing generation of residual strain anisotropy in the vertical or lateral directions of W-silicone sheets by controlling thermal exposure durations. (b) Photographs of W-silicone sheets heated for different time. Scale bars in all photographs are $5 \mathrm{~mm}$. When the time is $<4 \mathrm{~s}$, the Wsilicone sheet was folded in the patterned area. The folding angle increases as the heating time increases, indicating increased residual strain anisotropy. When the heating time is $>4 \mathrm{~s}$, the $\mathrm{W}$-silicone sheet will remain flat, suggest that the same physical property is realized in the whole sheet and no residual strain anisotropy was created.

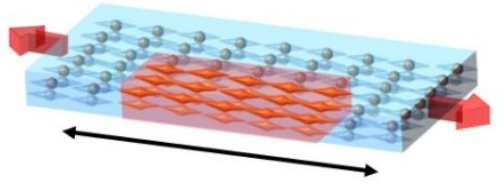

Lateral anisotropic residual strain
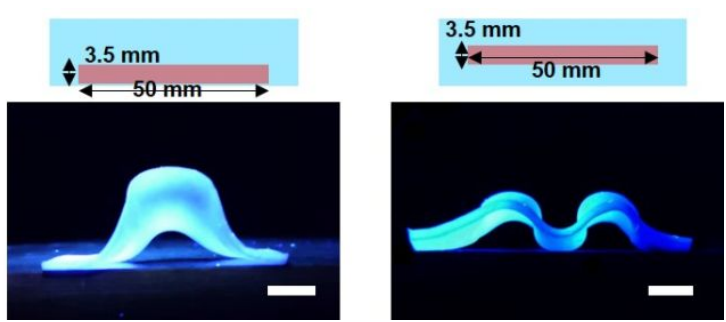

Figure S4. Photographs of 3D structures buckled from a W-silicone sheet. Scale bars are $5 \mathrm{~mm}$.
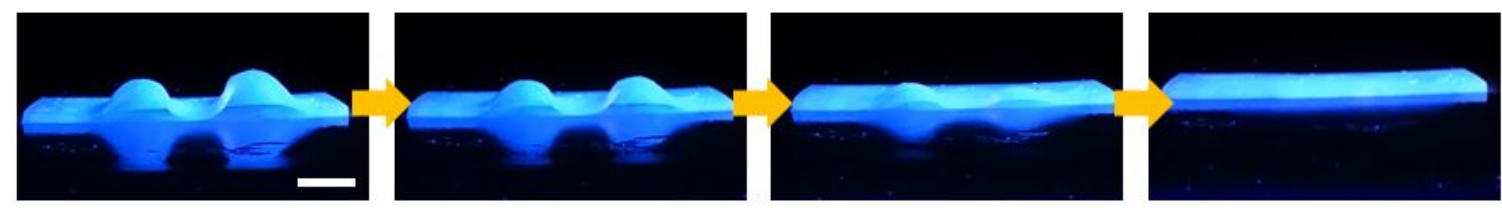

Figure S5. Photographs showing a shape recovering process of a buckled $\mathrm{W}$-silicone sheet on a $100{ }^{\circ} \mathrm{C}$ hotplate. Scale bar is $5 \mathrm{~mm}$. 

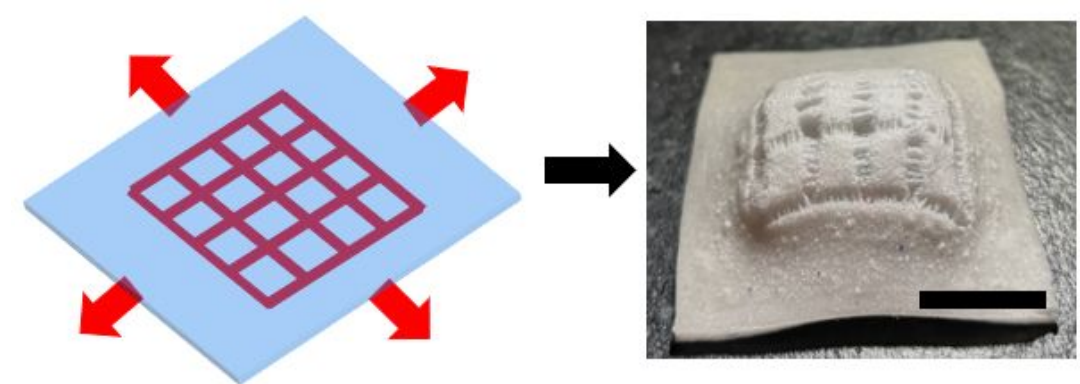

Figure S6. Photographs of 3D structures buckled from a W-silicone sheet ( $3 \mathrm{~cm} \times 3 \mathrm{~cm}$ x $1 \mathrm{~mm})$ by applying biaxial strain. Scale bar: $1 \mathrm{~cm}$.
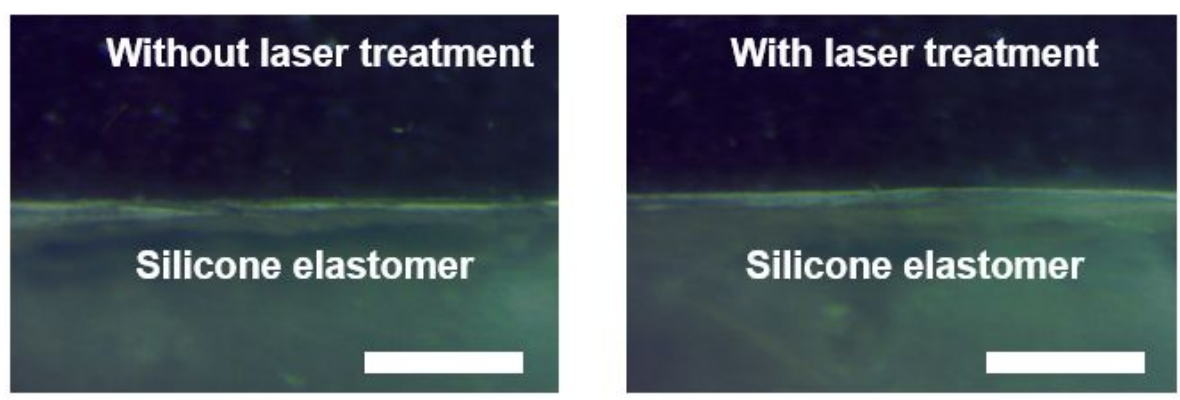

Figure S7. Cross-section optical images of pure silicone elastomer without and with laser irradiation. The elastomers sheet was $100 \%$ pretreated and then treated by a low-power laser, which does not ablate or damage the surface of the silicone elastomer. No surface wrinkle was formed on the pure silicone elastomer.

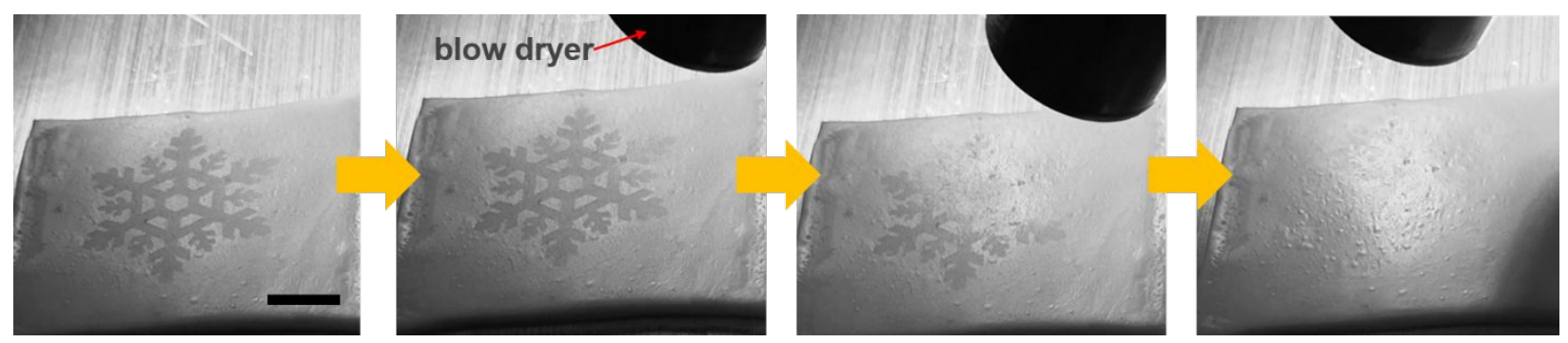

Figure S8. Photograph showing gradual disappearance of a wrinkle pattern on a W-silicone sheet by a blow dryer. Scale bar is $1 \mathrm{~cm}$. 


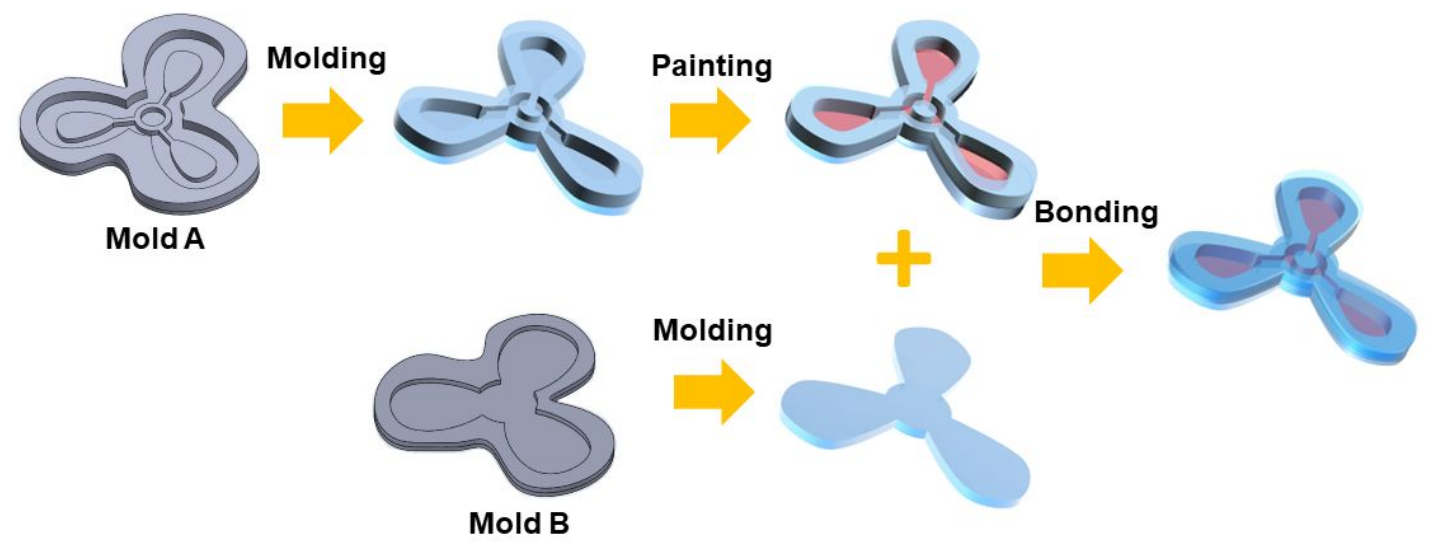

Figure S9. Schematic of fabricating a pneumatic actuator from W-silicone composites.

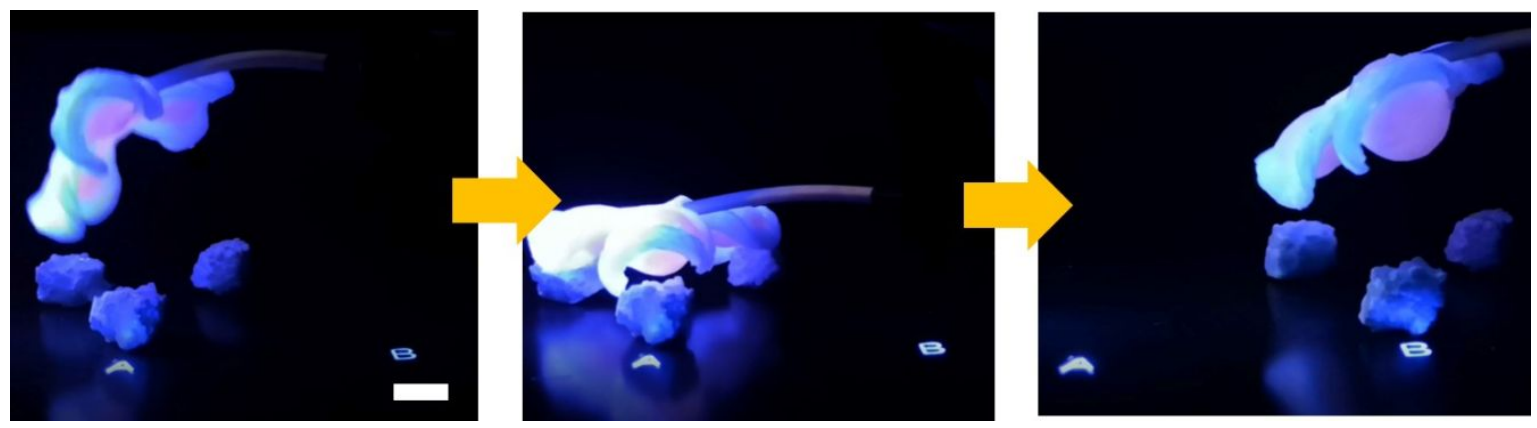

Figure S10. Photographs of a programmed pneumatic actuator grabs three plastic foam by three petals. Scale bar is $1 \mathrm{~cm}$. 


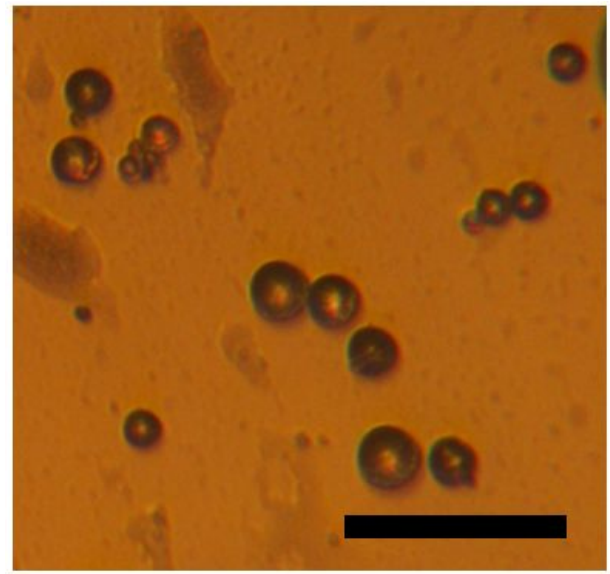

Figure S11. Optical image showing morphology of PCL MPs. Scale bar is $100 \mu \mathrm{m}$.

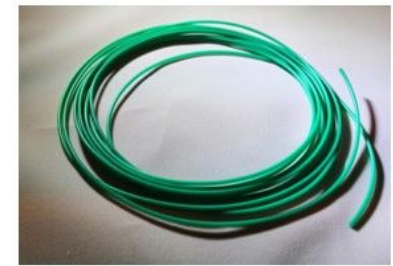

PCL filament

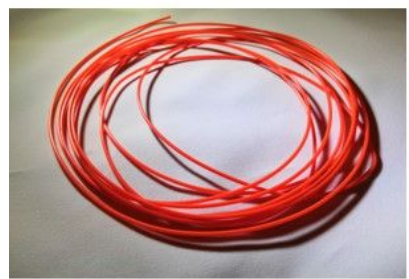

PCL filament

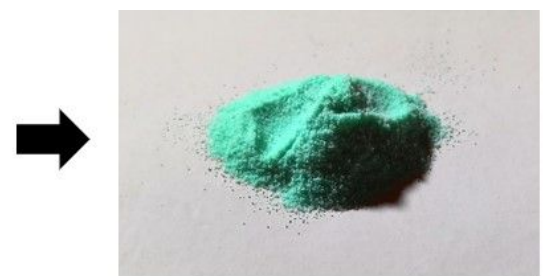

PCL MPs

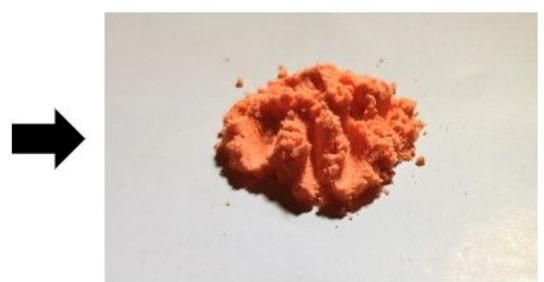

PCL MPs

Figure S12. Photograph of PCL filament and PCL MPs with different color. 


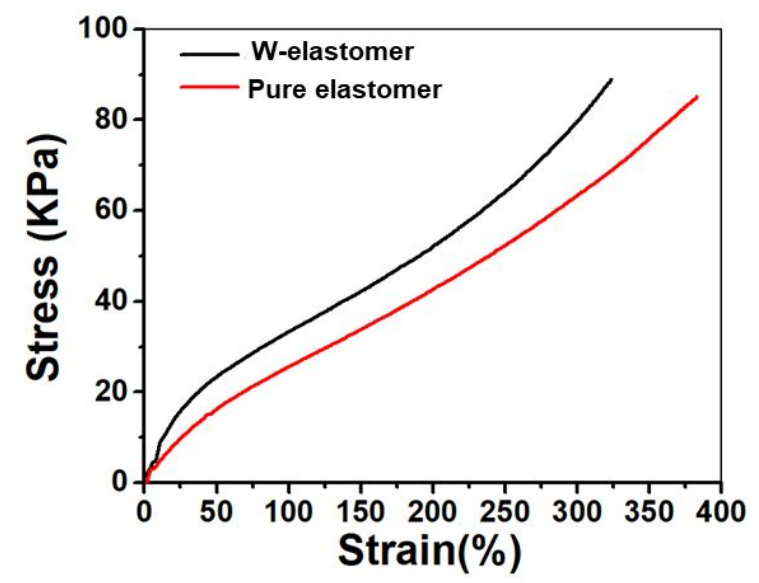

Figure S13. Stress-strain curves of W-silicone composites and pure silicone. 